\title{
Accuracy Improvement of the Alternating Current Zero Po- tential method for Impedimetric Sensor Matrices
}

\author{
Zheng $\mathrm{Hu}^{1}$, Dayu Chen ${ }^{1}$, Olfa Kanoun ${ }^{1}$ \\ 1 Technische Universität Chemnitz, Chemnitz, 09126, Germany \\ olfa.kanoun@etit.tu-chemnitz.de
}

\begin{abstract}
Summary:
In [1], we introduced the alternating current zero potential circuit (AC-ZPC) for two-dimensional resistive sensor matrices, where each matrix row is driven by an individual AC signal at a certain frequency, so that every column can be read separately. This method enables the simultaneous measurement of all sensors in the matrix and dispenses with multiplexers. In this work, a novel calibration method for the AC-ZPC for two-dimensional sensor matrices is proposed, where the measurement deviation caused by the used operational amplifiers has been eliminated. A measurement accuracy improvement has been thereby realized. This novel calibration strategy has been carried out by simulation for matrices of impedimetric sensors composed of one resistor in parallel to one capacitor. The achieved measurement accuracy regarding the capacitors is in the pico-farad range and is for the parallel resistors in the kiloOhm range.
\end{abstract}

Keywords: AC-ZPC method, impedimetric, sensor matrices, calibration, measurement deviation, impedance spectroscopy

\section{Background, Motivation an Objective}

The AC-ZPC method was proposed to overcome the crosstalk effect [1] in the two-dimensional sensor matrices. It has been proved to be very accurate for resistive sensor matrices. Its potential for impedimetric units has been also demonstrated [2].

As reported in [2], one of the measurement deviation sources in the AC-ZPC method is the output impedance of the voltage source connected to each matrix row. This effect was solved in [2]. An un-solved source of deviations is the nonideal properties of the used operational amplifiers [1, 2]. These result in an unsatisfactory deviation for impedimetric targets like the RC parallel pairs indicated in Table. 1. Especially the measurement results regarding capacitor values in the pico-farad range are worse than for parallel resistors in the kilo-Ohm range. This range is important for nanocomposite pressure [3] and strain sensors $[4,5]$ as well as temperature sensors [6].

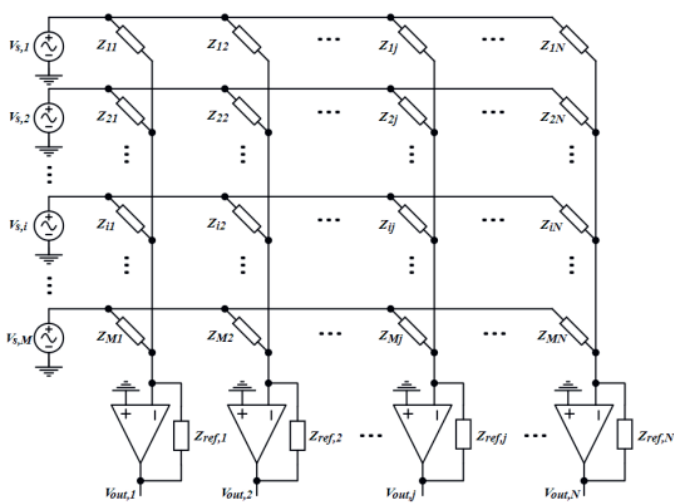

Fig. 1. The AC-ZPC method for $2 D$ sensor matrices of $M$ rows and $N$ columns.

In this work, a new calibration approach is proposed for correcting the deviations resulting from the non-ideal operational amplifiers, when the output impedance value of the row signal source can be ignored.

\section{Calibration method}

For matrices of $\mathrm{M}$ rows and $\mathrm{N}$ columns, when the voltage follower (s. Fig. 1) has been used for each voltage source $V_{s, i}$ for the $i^{\text {th }}$ row, its output impedance can be within $0.1 \Omega$ range, i.e., such small value can be ignored. Then the read-out voltage by the $j^{\text {th }}$ column operational amplifier

Table. 1. RC parallel pairs simulating the Impedimetric pressure sensor.

\begin{tabular}{lcccccccc}
\hline Applied weight $[\mathrm{N}]$ & 93.4 & 51.6 & 15.3 & 10.2 & 6.9 & 1.6 & 0.1 & 0.0 \\
\hline Resistance $[\mathrm{k} \Omega]$ & 1.9 & 2.9 & 11.0 & 15.9 & 22.6 & 48.6 & 75.1 & 100.4 \\
Capacitance $[\mathrm{pF}]$ & 361.7 & 300.4 & 149.6 & 102.0 & 75.8 & 22.8 & 10.0 & 7.3 \\
\hline
\end{tabular}


targeting the sensor on the $i^{\text {th }}$ row $Z_{i j}$ can be calculated as follows.

$$
\begin{aligned}
V_{\text {out }, j}\left\{1-\frac{1}{A_{j}}[1\right. & \left.\left.+Z_{\text {ref }, j}\left(\frac{1}{Z_{a m p, j}}+\sum_{m=1}^{M} \frac{1}{Z_{m j}}\right)\right]\right\} \\
& =-Z_{r e f, j} \frac{V_{s, i}}{Z_{i j}}
\end{aligned}
$$

$A_{j}$ and $Z_{a m p, j}$ are the finite gain factor and the input impedance of this op-amp, respectively. $Z_{m j}$ marks the other sensors connected to the same column but in different rows.

Based on this equation, we propose a new calibration method, where one extra row of given impedance value $Z_{\text {given }}$ is inserted into the sensor matrix: In the first phase, $V_{s, i}$ is connected to the $i^{\text {th }}$ row and the extra row is grounded. The resulting readout value by the $j^{\text {th }}$ column is named as $V_{\text {out }, j}^{i}$ here; In the second phase, $V_{s, i}$ is switched to the extra row, while the $i^{\text {th }}$ row is grounded. The resulting readout data by the same column is defined as $V_{\text {out }, j}^{\text {ext }}$. Both $V_{\text {out }, j}^{i}$ and $V_{\text {out }, j}^{\text {extra }}$ can be expressed using the same equation (s. above), then the expression of the targeted sensor can be recalculated.

$$
Z_{i j}=Z_{\text {given }} \frac{V_{\text {out }, j}^{\text {extra }}}{V_{\text {out }, j}^{i}}
$$

Hence, the unexpected deviation sources like $A_{j}$, $Z_{a m p, j}$ and $Z_{m j}$ have been calibrated out, and the measurement results regarding $Z_{i j}$ can be improved.

\section{Results}

The proposed calibration method has been simulated in LTspice for a $4 * 4$ sensor matrix, where the targeted sensor $Z_{11}$ varies as the values listed in Table. 1, and the other 15 sensors are $\mathrm{RC}$ pairs of $1.9 \mathrm{k} \Omega \| 361.7 \mathrm{pf}$ simulating the worse crosstalk effect. When the calibration method is carried out, an extra row of given $\mathrm{RC}$ pairs $(1.9 \mathrm{k} \Omega \| 361.7 \mathrm{pf})$ is added to this $4 * 4 \mathrm{ma}$ trix, to keep the consistency with other un-targeted sensors. The excitation signal for each row is a single sine wave having a frequency of $7 \mathrm{kHz}$, $11 \mathrm{kHz}, 17 \mathrm{kHz}$ and $23 \mathrm{kHz}$, respectively. Different voltage source impedance $Z_{r, i}$ has been also considered in the simulation.

As indicated in Fig. 3 and 4, the proposed calibration method performs always better than the non-calibrated one, facing different $Z_{r, i}$ values. In comparison to the original AC-ZPC method (the blue/red/yellow curves), the novel calibration method (the black/magenta/cyan curves) narrows the resistance measurement deviation within $\pm 0.1 \%$ range and suppresses the capacitance measurement deviation from more than $-3 \%$ to less than $1 \%$.

\section{References}

[1] Hu Z, Tan W, Kanoun O. High accuracy and simultaneous scanning AC measurement approach for two-dimensional resistive sensor arrays[J]. IEEE Sensors Journal, 2019, 19(12): 4623-4628.

[2] Hu Z, Ramalingame R, Kallel A Y, et al. Calibration of an AC Zero Potential Circuit for Two-Dimensional Impedimetric Sensor Matrices[J]. IEEE Sensors Journal, 2020, 20(9): 5019-5025.

[3] Ramalingame R, Hu Z, Gerlach C, et al. Flexible piezoresistive sensor matrix based on a carbon nanotube PDMS composite for dynamic pressure distribution measurement[J]. Journal of Sensors and Sensor Systems, 2019, 8(1): 1

[4] Sanli A, Müller C, Kanoun O, et al. Piezoresistive characterization of multi-walled carbon nanotube-epoxy based flexible strain sensitive films by impedance spectroscopy[J]. Composites Science and Technology, 2016, 122: 18-26.

[5] Bouhamed A, Al-Hamry A, Müller C, et al. Assessing the electrical behaviour of MWCNTs/epoxy nanocomposite for strain sensing[J]. Composites Part B: Engineering, 2017, 128: 91-99.

[6] Benchirouf A, Palaniyappan S, Ramalingame R, et al. Electrical properties of multi-walled carbon nanotubes/PEDOT: PSS nanocomposites thin films under temperature and humidity effects[J]. Sensors and Actuators B: Chemical, 2016, 224: 344-350.
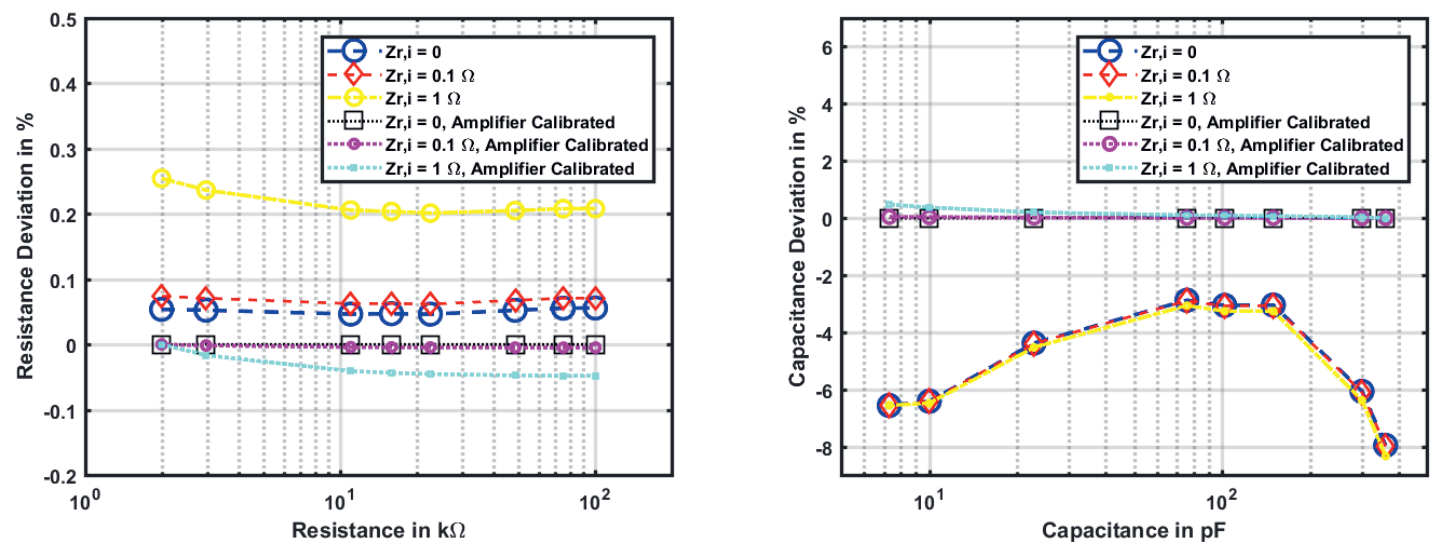

Fig. 3. Resistance and Capacitance deviation of the AC-ZPC method with/without calibrations. 